\title{
Mitochondrial Hsp90 chaperones as novel molecular targets in prostate cancer
}

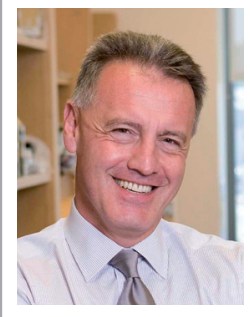

Dario C Altieri
"The goal of such 'network-oriented' drug discovery is to develop antagonists against so-called cancer 'nodal' proteins, molecules that integrate multiple signaling subnetworks, with the hope of simultaneously disabling more than one mechanism of disease maintenance."

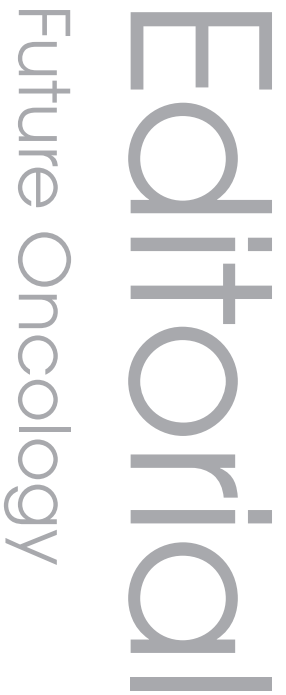

Prostate Cancer Discovery \& Development Program, Department of Cancer Biology, University of
Massachusetts Medical School, 364 Plantation Street, LRB428, Worcester, MA 01605, USA a Tel. +1 5088565775 m. Fax:+1 5088565792 m dario.altieri@umassmed.edu

Despite the controversies on how best to detect prostate cancer early, the survival rates of these patients have significantly improved over the last several years, and numerous options are available to manage early-stage disease. Unfortunately, what has changed little over the decades is the outlook of advanced prostate cancer, a disease stage characterized by resistance to androgen castration and tumor cell dissemination to distant organs, especially the bone. The therapeutic options for these patients are few and far between, systemic chemotherapy has limited efficacy, and the clinical outcome is invariably dismal, often with death from disease within 24 months from the onset of castration resistance [1]. The management of these patients remains a formidable challenge in the clinic, and constitutes an area in urgent need of fresh therapeutic approaches.

6....although feasible, in general,

Hsp90-based therapy proved to be disappointing in the clinic ... often at a cost of severe toxicity."

In this context, disparate agents targeting growth factor receptors, angiogenesis, the 'tumor microenvironment', anti-apoptotic mechanisms and cell adhesion, as well as enhancing antitumoral immunity, are being explored in patients with advanced prostate cancer (reviewed in [2]). Conversely, the extraordinary molecular and genetic complexity of advanced-stage disease suggests that targeting a single oncogenic pathway may not be sufficient to achieve durable remissions in these patients. Accordingly, novel drug discovery efforts have begun to exploit systems biology tools, and model cancer pathways as globally interconnected 'networks' [3]. The goal of such 'network-oriented' drug discovery is to develop antagonists against socalled cancer 'nodal' proteins, molecules that integrate multiple signaling subnetworks, hoping to simultaneously disable more than one mechanism of disease maintenance [4].

The molecular chaperone heat-shock protein-90 (Hsp90) is a well-known cancer nodal protein, orchestrating protein-folding quality control in multiple signaling pathways of cell proliferation, survival and adaptation [5]. These functions require the sequential ATPase cycles of the chaperone, and are dramatically exploited in cancer, where Hsp90 activity is potentially required to maintain the malignant phenotype [6]. Owing to its nodal properties, Hsp90 has been vigorously pursued as a target for network-oriented drug discovery [7], and several small molecule antagonists of its ATPase pocket are currently in advanced preclinical development or clinical evaluation. However, although feasible, in general, Hsp90based therapy proved to be disappointing in the clinic, certainly inferior to the expectation of global 'network inhibition', and achieving only modest patient responses, often at a cost of severe toxicity [8]. Clearly, significant gaps still exist in our understanding of Hsp90 that hamper its full exploitation for novel cancer therapeutics.

Recent data from our group may have helped bridge this gap, shedding new light onto a novel facet of Hsp90 biology in tumors; of potential importance in designing effective chaperonebased therapeutics. We found that Hsp90 and its related ATPase-directed chaperone, TNF receptor-associated protein-1 (TRAP-1), exist in a novel subcellular pool compartmentalized in the mitochondria of tumor cells, but not in most normal tissues in vivo [9]. In prostate cancer, TRAP-1 turned out to be a sensitive and easily accessible 
biomarker of disease progression, abundantly expressed in prostatic intraepithelial neoplasia, all Gleason grades of cancer, and metastatic disease to lymph nodes and bones, but was undetectable in normal prostatic epithelium or benign prostatic hyperplasia [10]. A similar paradigm was validated in genetic disease models in rodents, where TRAP-1 was abundantly found in prostatic lesions formed in transgenic adenocarcinoma of the mouse prostate (TRAMP) or Pten ${ }^{p c-1}$ mice [10], expressing the $S V 40$ oncogene, or lacking the $P T E N$ tumor suppressor in the prostate, respectively. The basis for the differential expression of mitochondrial Hsp90 chaperones in tumor versus normal tissues is not yet known. However, signaling pathways associated with cellular transformation may contribute to this response, as expression of an activated Ras oncogene in normal fibroblasts is sufficient to increase TRAP-1 levels in these cells, compared with nontransformed cultures [9].

Once in mitochondria, Hsp90 chaperones physically associate with the mitochondrial matrix immunophilin, cyclophilin D (CypD) [9]. This molecule is an integral component of the 'mitochondrial permeability transition pore', a multiprotein complex that controls organelle integrity and that, in response to cell death or stress stimuli, promotes a sudden increase in organelle conductance to solutes, that is, permeability transition pore opening [11]. In turn, this triggers a cascade of events characterized by dissipation of the mitochondrial inner membrane potential, swelling of the matrix, remodeling of the cristae and rupture of the outer membrane, driving the release of pro-apoptotic cytochrome $\mathrm{c}$ in the cytosol and the initiation of caspase-dependent apoptosis [11]. Data obtained from our laboratory have shown that mitochondrial Hsp90 chaperones vigorously oppose this process through a protein-foldingdependent mechanism, block CypD-dependent initiation of mitochondrial permeability transition and preserve organelle integrity against cell death stimuli [9]. In a teleological context, this global cytoprotective pathway appears ideally suited to maintain mitochondrial homeostasis in the face of the disparate stress conditions that invariably accompany tumor growth in vivo. Accordingly, forced expression of TRAP-1 in prostatic epithelial cells was sufficient to prevent caspase activation and block mitochondrial cell death in response to apoptotic stimuli, including taxanes, which are used as mainstay chemotherapy in patients with advanced disease [10].

Owing to their differential expression in transformed prostate as opposed to normal epithelium [10], and their role in a fundamental pathway of tumor cell survival [9], mitochondrial Hsp90 chaperones may provide an almost ideal therapeutic target in advanced prostate cancer. However, disabling this pathway for drug discovery proved challenging, as none of the small-molecule Hsp90 antagonists currently in the clinic has the ability to penetrate mitochondria [9,12]. To overcome these barriers, we recently synthesized GA mitochondrial matrix inhibitors (Gamitrinibs), the first class of subcellularly targeted Hsp90 ATPase antagonists designed to specifically inhibit the mitochondrial pool of the chaperone [12]. The structure of Gamitrinibs is combinatorial and comprises an ATPase-inhibitory module derived from 17-allylamino-demethoxygeldanamycin (17-AAG), a standard Hsp90 antagonist, fused to a mitochondriotropic moiety that directs it specifically to mitochondria. When tested against androgen receptor-negative prostate cancer cells, Gamitrinibs produced rapid and complete tumor cell killing [10], whereas $17-A A G$ was without effect. Mechanistically, the anticancer activity of Gamitrinibs reflects a sudden and irreversible collapse of mitochondrial integrity, mediated by CypD-dependent opening of a permeability transition pore [12]. Confirming their selective 'mitochondriotropic' design, Gamitrinib had no effect on Hsp90 homeostasis outside of mitochondria [12]. Importantly, normal prostatic epithelial cells were also insensitive to Gamitrinibs, and only forced expression of TRAP-1 in these cells made them susceptible to Gamitrinib-mediated killing [10].

"Mechanistically, the anticancer
activity of Gamitrinibs reflects a sudden
and irreversible collapse of
mitochondrial integrity..."

Altogether, these results open intriguing possibilities for novel cancer drug-discovery platforms, especially when it comes to currently intractable tumors, such as advanced and metastatic prostate cancer. Although a broader approach of network-oriented drug discovery is probably best suited to conquer these highly heterogeneous malignancies [4], it is clear from the data mentioned above that the development of such network inhibitors must take into consideration the spatial subcellular compartmentalization of the target. Signaling networks are not arranged randomly in cells, especially in deranged tumor cell types, but rather orchestrated in specialized and semi-autonomous subcellular compartments, ideally suited to impart additional functionality and adaptability for tumor growth. In the case of 
Hsp90, this subcellular segregation in mitochondria has critical implications for tumor cell survival and, potentially, for the regulation of sensitivity to Hsp90-based therapeutics [9]. Whether the inability of nonsubcellularly targeted $\mathrm{Hsp} 90$ antagonists to penetrate mitochondria may explain, at least in part, their modest activity in the clinic, is currently unknown [8]. However, it is intriguing that Gamitrinibs possess a much broader spectrum of anticancer activity than their nonsubcellularly targeted counterparts, indistinguishably killing 'sensitive' or 'resistant' tumor cell types regardless of genetic makeup, loss of tumor suppressors or expression of survival pathways that often drive treatment failure [12]. With respect to advanced prostate cancer, Gamitrinibs may prove useful against metastatic disease, given the abundance of mitochondrial Hsp90s in disseminated lesions, including bones [10]. Despite the universal role of mitochondria in cellular homeostasis, 'mitochondriotoxic' cancer therapy with Gamitrinibs was surprisingly well tolerated, at least in preclinical models, with no evidence of systemic or organ toxicities [12]. Such a desirable therapeutic window may reflect not only the sharp differential expression of the targets, that is, mitochondrial Hsp90s, in tumors compared with normal cells [9], but also the approximately 100-fold higher affinity with which Hsp90 in tumors binds small-molecule ATPase inhibitors, including Gamitrinibs, as opposed to normal tissues [13].

Clearly, several questions remain to be answered to further credential mitochondrial Hsp90s and their subcellularly targeted inhibitors for a novel paradigm of 'compartmentalized networkoriented drug discovery'. For instance, we do not know whether mechanisms of resistance will eventually limit the efficacy of Gamitrinibs or that of other comparable subcellularly targeted Hsp90 inhibitors, in vivo. So far, no tumor cell types resistant to Gamitrinibs have been found, but the possibility still exists and must be thoroughly investigated in future studies. We also do not know whether Gamitrinibs will have adequate druglike properties to support their clinical development for testing in humans. Finally, although promising in preclinical studies, the potential for unforeseen toxicities with these agents, especially after prolonged treatment, remains to be carefully evaluated. For now, available evidence points to mitochondrial Hsp90 chaperones as novel and potentially attractive therapeutic targets in advanced prostate cancer [10], and identifies their subcellularly directed antagonists, that is, Gamitrinibs [12], as a conceptually novel class of anticancer agents, worthy of further preclinical and clinical investigation.

\section{Financial \& competing interests disclosure \\ This work was supported by NIH grants CA90917, CA78810 and CA118005. The author has a filed pat- ent currently pending on the structure and utility of Gamitrinibs as anticancer agents. The author has no other relevant affiliations or financial involvement with any organization or entity with a financial interest in or financial conflict with the subject matter or materials discussed in the manuscript apart from those disclosed. No writing assistance was utilized in the production of this manuscript.}

\section{Bibliography}

1. Berthold DR, Pond GR, Soban F et al: : Docetaxel plus prednisone or mitoxantrone plus prednisone for advanced prostate cancer: updated survival in the TAX 327 study. J. Clin. Oncol. 26, 242-245 (2008).

2. Taichman RS, Loberg RD, Mehra R et al.: The evolving biology and treatment of prostate cancer. J. Clin. Invest. 117, 2351-2361 (2007).

3. Araujo RP, Liotta LA, Petricoin EF: Proteins, drug targets and the mechanisms they control: the simple truth about complex networks. Nat. Rev. Drug Discov. 6, 871-880 (2007).

4. Butcher EC: Can cell systems biology rescue drug discovery? Nat. Rev. Drug Discov. 4, 461-467 (2005).

5. Zhao R, Houry WA: Molecular interaction network of the Hsp90 chaperone system. $A d v$. Exp. Med. Biol. 594, 27-36 (2007).
6. Whitesell L, Lindquist SL: HSP90 and the chaperoning of cancer. Nat. Rev. Cancer 5 , 761-772 (2005).

7. Isaacs JS, Xu W, Neckers L: Heat shock protein 90 as a molecular target for cancer therapeutics. Cancer Cell 3, 213-217 (2003).

8. Drysdale MJ, Brough PA, Massey A et al.: Targeting Hsp90 for the treatment of cancer. Curr. Opin. Drug Discov. Devel. 9, 483-495 (2006).

9. Kang BH, Plescia J, Dohi T et al.: Regulation of tumor cell mitochondrial homeostasis by an organelle-specific Hsp90 chaperone network. Cell 131, 257-270 (2007).

10. Leav I, Plescia J, Goel HL et al.: Cytoprotective mitochondrial chaperone TRAP-1 as a novel molecular target in localized and metastatic prostate cancer. Am. J. Pathol. 176, 393-401 (2010).

11. Green DR, Kroemer G: The pathophysiology of mitochondrial cell death. Science 305 , 626-629 (2004).
12. Kang BH, Plescia J, Song HY et al.: Combinatorial drug design targeting multiple cancer signaling networks controlled by mitochondrial Hsp90. J. Clin. Invest. 119, 454-464 (2009).

13. Kamal A, Thao L, Sensintaffar J et al.: A high-affinity conformation of $\mathrm{Hsp} 90$ confers tumour selectivity on Hsp90 inhibitors. Nature 425, 407-410 (2003).

\section{Affiliation}

- Dario C Altieri, MD

Prostate Cancer Discovery \& Development Program, Department of Cancer Biology, University of Massachusetts Medical School, 364 Plantation Street, LRB428, Worcester, MA 01605, USA

Tel. +15088565775

Fax: +1 5088565792 dario.altieri@umassmed.edu 